\title{
Variations
}

Variations

Revue internationale de théorie critique

$17 \mid 2012$

Critique du travail

\section{Travail, sexe et État. Une démarche anthropologique}

Laurent Bazin et Monique Selim

\section{(2) OpenEdition}

Journals

Édition électronique

URL : http://journals.openedition.org/variations/360

DOI : 10.4000/variations.360

ISSN : 1968-3960

Éditeur

Les amis de Variations

Référence électronique

Laurent Bazin et Monique Selim, «Travail, sexe et État. Une démarche anthropologique », Variations [En ligne], 17 | 2012, mis en ligne le 15 octobre 2012, consulté le 02 mai 2019. URL : http:// journals.openedition.org/variations/360 ; DOI : 10.4000/variations.360

Ce document a été généré automatiquement le 2 mai 2019.

Les ami•e•s de Variations 


\title{
Travail, sexe et État. Une démarche anthropologique
}

\author{
Laurent Bazin et Monique Selim
}

\section{NOTE DE L'AUTEUR}

Cet article est le fruit d'une réflexion et de recherches de terrain menées dans le cadre du projet ANR-10-Suds-010-01 intitulé CRITERES (Crises - Travail, emploi, revenus dans les pays du Sud). Nous tenons à remercier Pascale Absi pour sa lecture attentive.

1 La « crise des subprimes » en 2007-2008 puis la « crise de la dette » qui s'amorce en 2011 ont contribué à replacer l'attention médiatique, en Europe, sur la question du travail, de sa précarisation et du démantèlement de ses protections. Ce regain d'actualité se traduit par une multiplication des publications scientifiques sur le travail ces dernières années, qui comportent une dimension "genrée » de plus en plus présente. Le travail, comme objet scientifique, avait en effet été relativement éclipsé dans les années 1990, le débat philosophique sur la « fin du travail » se présentant précisément comme le symptôme de cette élision. Un «retour sur la condition ouvrière» (Beaud, Pialoux, 2005) et sur l'observation du travail (Arborio et alii, 2008) ${ }^{1}$ s'opère, pour mettre en évidence notamment la fragilisation des statuts d'emploi dont sont victimes en premier lieu les femmes, les nouvelles formes de domination dans le travail ${ }^{2}$, ainsi que la souffrance (Dejours, 2000) et les résistances qu'elles suscitent.

2 L'accroissement colossal des inégalités au niveau mondial en constitue un décor, peu articulé avec la question du travail, sinon dans son aspect global, statistique, de la répartition des richesses et des revenus. En revanche, à travers le prisme du genre, l'inégalité homme/femme perdurante est devenue une sorte de parangon des inégalités et des discriminations.

3 Les délocalisations, la mondialisation des processus productifs, la concurrence internationale exacerbée autour de la question du « coût du travail », les succès des pays 
émergents - comme les dragons asiatiques vingt ans auparavant - font porter l'intérêt, avec souvent de grandes confusions, sur la différenciation des modalités du travail dans les diverses régions du monde. Peu de travaux scientifiques, cependant, mettent en œuvre une véritable perspective comparative. D'un autre côté, le cycle de l'endettement des États et des ajustements structurels, qui s'ouvre en Afrique dans les années 1980 pour se refermer sur l'Europe en 2010, rappelle plus que jamais qu'on ne peut raisonner sur une partition nord/sud du monde. Un comparatisme rigoureux invite à réfléchir à la fois sur l'unicité des processus et leur singularité dans chaque contexte, dans chaque situation. Ces remarques soulignent l'intérêt d'une approche anthropologique du travail - qui se situe à l'opposé d'une optique ethnographique (Bazin, Selim, 2002) - capable d'analyser précisément les rapports de travail en les replaçant dans leur architecture sociale, d'en appréhender les transformations et en les situant par rapport aux dynamiques sociales et politiques propres à chaque société, ainsi qu'aux processus globaux auxquelles elles sont confrontées.

4 Les crises récentes apparaissent comme autant de moment où l'État se révèle un acteur clé du travail, et le travail une question politique centrale. Si la puissance publique est au cœur de la répartition des richesses et de leur partage entre capital et travail, si son rôle est essentiel dans la régulation des systèmes de protection sociale, la place de l'État dans le travail ne se réduit cependant pas à la gestion des équilibres économiques. Pour le faire apparaitre clairement, il convient d'examiner dans chaque contexte socio-historique, les modes spécifiques d'édification de l'État, ainsi que la manière dont la figure imaginaire de l'État s'inscrit dans les configurations du travail.

5 Nous proposons dans cet article un passage en revue épistémologique de cette question sur un double niveau. Dans un premier temps, il s'agira de resituer rapidement la manière dont l'État et le travail sont liés dans les paradigmes des sciences sociales, pour montrer l'évolution des optiques scientifiques et proposer une approche anthropologique mettant en lumière l'importance de la figure de l'État dans les rapports de travail. Dans un second temps, nous nous attacherons plus spécifiquement à examiner le développement récent d'une problématique "genre et travail ", pour montrer là encore l'oubli tendanciel de l'État. Les perspectives proposées seront illustrées par des travaux réalisés par les auteurs en Algérie et en Chine.

\section{État et travail}

6 Quelles que soient les divergences théoriques, par exemple entre courants marxistes et libéraux, la relation État-travail est abordée classiquement à partir du rôle central de l'État dans l'émergence du salariat dans les pays industrialisés européens et dans le traitement de la question sociale. Historiquement, en effet, au cours du processus d'industrialisation en Europe et aux États-unis du XVIII ${ }^{e}$ au XX ${ }^{e}$ siècles, l'État par son pouvoir législatif, de contrainte et de répression, est l'acteur central de la destruction des anciens cadres qui régissaient le travail (les corporations, le servage, l'esclavage, le travail forcé, etc.), de la mise en place d'un marché du travail «libre » devant gouverner les rapports entre une « demande » (les entreprises) et une " offre » (les travailleurs), puis de l'élaboration progressive d'une protection garantissant à la fois un cadre légal au contrat de travail et des dispositifs de sécurité sociale. En retour, l'État lui-même a été durant deux siècles un enjeu central de la lutte des classes de sorte que, de révolutions en réformes, sa nature contemporaine peut être considérée en grande partie comme le 
produit des conflits du travail et de la nécessité de leur régulation. Peu d'attention est accordée par les sciences sociales, dans leurs paradigmes dominants, à ce qui se passe en dehors des pays industrialisées. Pourtant, la question du travail a été un problème constant dans les Empires coloniaux, depuis leur constitution jusqu'à la décolonisation (Cooper, 1996). De plus, les luttes politiques et idéologiques autour de la structuration de l'État par le travail, et du travail par l'État, se sont internationalisées et ont dominé toute une moitié du XXe siècle, scandées par les grandes mutations de l'ordre géopolitique du monde : la révolution d'octobre en Russie, la crise des années 1930, la seconde guerre mondiale, la décolonisation de l'Afrique et de l'Asie, la guerre froide, enfin, ont entraîné la formation d'une multiplicité de modèles d'États qui sont autant de tentatives de construire le politique et la société autour de la mise en ordre de la relation État-travail : États socialistes, fascistes (Europe), péronistes et populistes (Amérique latine), dictatures anti-communistes, États dits " providence » (Europe), ou modèles hybrides expérimentés à la périphérie.

7 En dépit de leur faible intérêt pour les trois quarts du monde, les grands courants de pensée et les débats théoriques qui les opposent sont, jusque dans le dernier quart du $\mathrm{XX}^{\mathrm{e}}$ siècle, en majeure partie fondés sur leur positionnement vis-à-vis de la relation entre l'État et le travail, le marxisme occupant une place prépondérante. Quelles que soient les options théoriques, le travail s'impose comme une question politique. Que l'État soit un acteur essentiel dans la configuration des rapports de travail est donc une réalité bien ancrée dans l'histoire de toutes les disciplines des sciences sociales.

8 Cette question a pourtant été progressivement éclipsée dans les années 1980, à la faveur de l'affaiblissement du socialisme et de la révolution néolibérale qui abolissent les alternatives au capitalisme, ce dernier étant lui-même désigné désormais comme " économie de marché ", dont le modèle se répand à son tour dans le monde entier. Tout se passe comme si la valorisation du marché éclipsait désormais la référence au travail ${ }^{3}$. Notons que dans le même temps, cette mondialisation du marché se traduit par une reconfiguration politique autour d'idéologies de l'identité nationale dont se dotent les États contemporains (Bazin, Gibb, Selim, 2007). Elles ont pour effet de redéfinir le travail sur une base nationale, en excluant les migrants ou des groupes minoritaires de l'accès à certaines catégories d'emploi, selon les situations.

9 Comment expliquer cette évolution épistémologique? Dans les pays occidentaux, la stabilisation du salariat a été suffisamment bien institutionnalisée dans les périodes antérieures pour ne plus être problématisée comme une question politique, au sens fort du terme : elle devient une question de politique publique, d'autant que les problèmes de chômage structurel et de flexibilisation du travail commencent alors à se poser. La sociologie du travail, qui s'est historiquement constituée dans une alliance symbolique avec les mouvements syndicaux et le monde du travail, doit faire une place à la sociologie des professions, ou à celle de l'entreprise. Cette dernière se rapproche des perspectives managériales sur l'organisation du travail, la conduite du changement, la «culture d'entreprise ».

Dans les pays dits en développement, les problématiques se posent sous un angle différent, du fait de la disjonction entre emploi salarié et travail, que la sociologie française fera mine de "redécouvrir » au tournant des années 2000. Dans ces contextes, c'est la question de l'informel qui va dominer les débats à partir de la seconde moitié des années 1970, mettant l'accent sur le manque d'État, ou ses défaillances dans l'institutionnalisation du travail. Ces débats, quels que soient les défauts conceptuels 
qu'ils contiennent, mettent l'accent en creux sur le fait que l'État définit les normes du travail, quand bien même il s'avère dans l'incapacité - ou montre l'absence de volonté de leur mise en œuvre (Lautier, Miras, Morice, 1994). Plus qu'un véritable concept, dont la définition échappe constamment, l'informel est une sorte de métaphore qui porte dans les représentations l'écart entre une norme idéelle et la réalité des rapports sociaux et économiques. Entre modèles d'État et modèles du travail s'insinue l'informel qui opère comme un jeu de faux-semblants : le travail trahit les carences de l'État tandis que l'État dénonce les carences du travail.

Qu'en est-il des tendances actuelles dans l'appréhension de la relation État-travail ? Les sciences économiques n'ont jamais perdu de vue trois aspects décisifs : l'État intervient dans la régulation du conflit capital-travail, et l'arbitrage de la répartition des valeurs ajoutées entre le capital et le travail ; son rôle se situe d'une part dans la répartition des richesses et d'autre part dans la régulation des marchés, dont celui du travail (dans les modèles actuels de l'«économie de marché ", la régulation monétaire privilégie la stabilisation des marchés financiers, au détriment du marché du travail, qui n'est plus qu'une "variable d'ajustement »); enfin, l'État agit au niveau des politiques publiques qu'il met en œuvre (politique d'emploi, mais aussi plus globalement politique économique, sociale, d'enseignement et de formation, etc.). Au cours des deux dernières décennies, après la vague des ajustements structurels ultralibéraux en Afrique et Amérique latine, l'influence de la banque mondiale a été capitale dans la reformulation des problématiques, appuyant autant l'évacuation du politique que celle du travail, avec les notions centrales de «gouvernance » et de " réduction de la pauvreté ». L'État devient un bon ou mauvais gestionnaire tandis que l'attention est portée sur les "pauvres", notamment la masse de ceux qui n'ont pas accès à un « emploi décent » (OIT). Les théories néolibérales croisent celles du «capital social» pour redéfinir tendanciellement les chômeurs comme entrepreneurs, porteurs d'un capital personnel qu'un accès au microcrédit pourrait faire fructifier, dissimulant ainsi le chômage, le sous-emploi et l'exploitation dans un scénario qui constitue le simulacre du rapport entre entreprises et marchés financiers dans le capitalisme. De ce nouveau décor idéologico-scientifique, l'État disparaît tout autant que le travail.

Dans une partie de la sociologie, tout comme en économie, demeure la conception de l'État comme arbitre du jeu social, entre les employeurs et les salariés. L'État dispose pour cela d'un ensemble d'instruments en termes juridiques et de politiques publiques : droit du travail ; arbitrage du rapport entre les "partenaires sociaux »; politique d'emploi ; garantie de l'assurance sociale (chômage, retraite); politique sociale et définition des minima sociaux.

Une approche sociologique plus récente, qu'on pourra désigner comme constructiviste, examine l'État comme force instituante. À travers ses politiques d'emploi, l'État contribue à définir le travail, l'emploi, le chômage. Par leur existence même ces politiques instaurent des normes de l'emploi tandis qu'elles laissent dans l'ombre certaines formes de travail (travail au noir, informel, domestique) ou l'attribuent à certaines propriétés sociales. De nombreux travaux ont été réalisés par la sociologie du travail en Europe et aux États-unis, pour montrer comment la notion même de population active, ainsi que les statuts de l'emploi et du chômage, émergent avec le développement du système salarial et se définissent à travers les politiques de l'emploi. Une telle problématisation des notions d'emploi, de chômage - ou de sous-emploi - est plus ancienne dans des sociétés caractérisées par un moindre degré de généralisation du travail salarié, et où par 
conséquent les catégories d'emploi et de chômage s'avèrent bien plus bien plus en décalage avec la réalité (Althabe, 1963), mais elles ont peu influencé les paradigmes dominants des sciences sociales.

Dans un contexte de mondialisation marqué depuis une trentaine d'année par une libéralisation des économies entraînant une flexibilisation accrue du travail, la mise en œuvre de politiques publiques de l'emploi se généralise, un temps déterminée par les dispositifs de «lutte contre la pauvreté ». Ces politiques adoptent, selon les contextes nationaux, des formules originales ou inspirées de modèles standardisés: microentreprise et auto-entrepreneuriat, recours au microcrédit, travaux d'utilité publique correspondant à une logique du workfare (par opposition au welfare), emplois subventionnés par la puissance publique, etc. En associant certaines caractéristiques à la reconnaissance de l'état de "chômeur", en ciblant leurs actions sur des publics spécifiques (les jeunes, les femmes, les vieux, les diplômés, les non-diplômés, etc.) et en les orientant vers des formes d'emploi particulières, ces politiques publiques contribuent à redéfinir les normes du travail, du chômage et de l'emploi : elles produisent et véhiculent à leur tour des représentations du travail qui amplifient les effets de reconfiguration du marché du travail.

\section{Figures de l'État dans le travail}

L'accent mis sur les politiques publiques et leur caractère instituant risque toutefois d'oblitérer les interrogations portant sur l'importance de la nature de l'État et de ses modes d'intervention dans la configuration des mondes du travail. Pour le dire autrement en utilisant la distinction bien commode de la langue anglaise, le politique (politics) n'est pas qu'une affaire de politiques (policies) et c'est un contresens que de réduire l'État à ses seules capacités d'action ou à la somme de ses administrations. Si l'État est une puissance agissant au moyen de dispositifs concrets ou formels, il est aussi un acteur symbolique central des sociétés contemporaines. Les recherches anthropologiques sont à peu près les seules à envisager dans leurs analyses la manière dont la figure imaginaire et symbolique de l'État s'internalise dans les situations de travail. Les voies en sont multiples, et se présentent comme singulières dans chaque société. Elles dépendent de la manière dont l'État s'est historiquement édifié, du mode d'instauration de son autorité et de ses assises politiques. Elles dépendent aussi de la manière dont les individus et les groupes sociaux articulent dans leurs itinéraires et leurs représentations les différents champs sociaux du travail, de la famille, du politique, du religieux; comment ils se positionnent face aux différentes formes d'autorité issues de ces différents champs, surplombées par la figure de l'État, représentation de l'autorité par excellence.

En Algérie, par exemple, les représentations du travail demeurent marquées par l'expérience de l'industrialisation massive qui a culminé dans les années du "socialisme » des années 1970 pour être progressivement démantelée dès la décennie suivante. L'État paraît aujourd'hui d'autant plus en dette vis-à-vis de la société qu'il se maintient dans un rôle d'assistance et de redistribution, sans être en mesure de s'acquitter de la fonction qu'il s'assigne, tandis la cupidité des dirigeants et leur accaparement des richesses sont sans cesse dénoncés. La demande d'emplois et de logements constitue aujourd'hui un ressort essentiel du rapport à l'État, qui se dramatise dans des actions désespérées ou des révoltes épisodiques (émeutes, groupes de protestataires, suicides publics par le feu, automutilations), le phénomène de la harga $a^{4}$ ou encore dans une multiplication des 
grèves faisant appel à l'autorité du président. En 2011, face aux « révolutions arabes », la réponse politique du gouvernement enrichi par la hausse de la rente pétrolière consiste à faciliter les aides à la création d'entreprise (crédit et microcrédit), dont sont chargées les agences pour l'emploi et la caisse d'allocation chômage créées pour amortir les licenciements massifs du secteur public des années 1994-97; elle mise aussi sur une injection massive de fonds dans les entreprises publiques ou privées afin d'en assainir la comptabilité.

17 Les représentations du travail sont l'objet de tiraillements et de contradictions qui reflètent les tensions sociales et en constituent un révélateur. Malgré une diminution drastique du taux de salariés dans la population active, le seul travail qui soit considéré comme un "vrai» travail demeure, dans les fractions sociales populaires, l'emploi déclaré dans le secteur public. Ce n'est ni l'occupation ni le revenu qui définissent le travail, mais «l'assurance » (sociale) et la stabilité de l'emploi. Ces représentations se fondent moins sur des avantages matériels ou sur la réalité d'une protection sociale que sur la recherche d'une reconnaissance et le sentiment d'une obligation pour l'État d'assurer la prise en charge des citoyens. L'emploi informel, dont doivent se contenter une majorité de la population, peut ainsi donner lieu à un ressentiment vis-à-vis de la puissance publique: "l'État n'a rien fait pour nous " ${ }^{5}$. A cet imaginaire répond un discours négatif omniprésent que tiennent les classes sociales moyennes et supérieures, ou encore les émigrés, répétant à l'envi que "les Algériens » ne veulent pas travailler, qu'ils ne savent pas travailler, qu'ils travaillent peu et lentement. Appuyées par des déclarations officielles du chef de l'État, ces représentations négatives s'alimentent depuis une dizaine d'années de la présence de nombreuses firmes chinoises opérant dans le secteur du bâtiment et des travaux publics, venues avec leurs propres ouvriers, réputés à l'inverse efficaces et productifs.

18 À un troisième niveau, les représentations du travail traduisent aussi des tensions qui traversent autant la différenciation sexuelle que les rapports familiaux dans leur ensemble. Les rapports sociaux de sexe, en particulier, sont l'objet de tiraillements multiples stimulés par la réislamisation de la société engagée dans les années 1980 et les femmes détentrices d'un emploi ont été particulièrement ciblées par des agressions durant le conflit des années 1990 entre le pouvoir et les factions islamistes, comme tous les acteurs symbolisant une position "progressiste». Si, en Algérie comme dans de nombreuses régions du monde, la majorité des étudiants sont désormais des femmes, le taux d'activité féminine stagne néanmoins à un niveau parmi les plus faibles du monde. Les ouvriers du bâtiment rencontrés dans les enquêtes de terrain menées à Oran estiment parfois qu'une « vraie femme » ne travaille pas hors de la maison, tandis que leur virilité ( rajla, de rajel: homme; le terme traduit aussi la notion d'honneur) dépend de leur capacité à entretenir leur famille et leurs dépendants : de leur point de vue, l'emploi est avant tout un attribut masculin. Le taux élevé du chômage et la sévère "crise du logement " se répercutent sur les rapports familiaux, obligent à la cohabitation entre générations dans des habitations trop exiguës, rendent problématique la conclusion des mariages et freinent l'émancipation des jeunes adultes. Un désespoir tangible s'exprime dans les entretiens avec de jeunes hommes à l'emploi instable, qui imaginent comme seul salut de traverser la Méditerranée, moins pour échapper à l'indignité de leur condition, que pour la vivre loin des regards que la société porte sur eux.

Si le manque de logements et d'emplois alimente les tensions politiques et constitue un pivot du rapport à l'État, c'est précisément que l'accès à ces deux ressources se situe au 
cœur des enjeux de la reproduction sociale et de la sexuation des rapports sociaux. Ces pressions sur les rapports familiaux sont en retour projetées sur l'État lorsqu'on lui reproche de ne pas s'acquitter correctement de ses obligations envers les citoyens, qui figurent ainsi comme des dépendants symboliques. Le terme hogra, omniprésent dans le lexique politique des médias d'opposition, traduit cette dimension conflictuelle du rapport à l'État. Sous ce vocable sont dénoncés le mépris et la maltraitance des citoyens ainsi que l'indignité que ces derniers ressentent en retour, sur des sujets qui s'étendent pêle-mêle des excès d'autoritarisme et de la violence du pouvoir jusqu'au chômage et à l'absence de logements disponibles.

Quelques résultats d'une enquête ethnologique en cours dans une entreprise publique du bâtiment à Oran, permettront enfin d'illustrer comment la figure imaginaire de l'État s'internalise dans les rapports de travail. Issue en 1997 du démantèlement de sociétés d'État prestigieuses dans les années 1970-80, la firme apparaît durant l'été 2011 dans une situation d'abandon: aucun investissement n'a été réalisé depuis sa création et les commandes ne sont guère suffisantes pour occuper pleinement les journées de travail. Lors des entretiens, les cadres font montre d'une grande nostalgie de la période socialiste qu'ils caractérisent par la grandeur de ses réalisations architecturales, la performance de la formation professionnelle et les avantages accordés au personnel. En revanche, on ne trouve guère de trace d'une nostalgie du passé dans les paroles des ouvriers, nulle évocation d'avantages perdus, mais plutôt le regret d'une diminution de leurs revenus, signe de la dévalorisation de leur travail et de leur statut. Leurs propos sont orientés vers une dénonciation virulente de la direction et des cadres, qu'ils accusent d'une corruption dont l'ampleur menacerait la capacité de redressement et de développement de l'entreprise. La composition du comité syndical conserve - selon un schéma répandu dans tout le tissu industriel - une structure héritée du système de parti unique et calquée sur les dispositifs de contrôle que l'État introduit à tous les échelons de la société, qui assure la sujétion politique tout en protégeant la corruption : l'affiliation obligatoire à un syndicat unique dont le représentant est simultanément membre de la direction et, ici, responsable de la sécurité. La structure des rapports hiérarchiques qui se reflète dans les entretiens menés présente deux caractéristiques : une capture du pouvoir par les réseaux d'intérêts, d'alliance et de dépendance qui a pour enjeu de s'accaparer des ressources et de neutraliser la contestation ; un rapport conflictuel entre les deux strates des cadres et des ouvriers. Ce dernier point fait écho aux situations mises en évidence par certains sociologues algériens vers la fin des années $1980^{6}$. Leurs explications mettaient l'accent sur les conflits entre fractions des classes dirigeantes, les contradictions et les erreurs de l'industrialisation volontariste, le décalage entre la logique industrielle des ingénieurs et les caractéristiques socio-culturelles des ouvriers algériens. Ces derniers seraient notamment porteurs d'un égalitarisme encouragé par le "populisme" de l'État, contredisant les hiérarchies professionnelles. Les matériaux recueillis vingt ans après ces travaux pionniers orientent vers d'autres interprétations : ce qui apparaît en jeu dans ce rapport conflictuel, c'est la réplication dans l'entreprise de la structure du pouvoir politique, fondé sur le contrôle, les relations de clientèle et l'accaparement des richesses. Ce constat ne peut cependant être simplement projeté rétrospectivement sur les situations passées pour les réexaminer : elle traduit en effet une certaine représentation de la conjoncture actuelle, où la puissance économique et financière de l'État est passée, au moins partiellement, dans les réseaux des factions qui le contrôlent, où la volonté modernisatrice de l'État a cédé la place devant des formes diverses d'exploitation; la construction nationale, autrefois emblématisée dans les aménagements et les réalisations 
architecturales des entreprises publiques, apparait aujourd'hui menée par des firmes étrangères et l'État semble de tout point de vue défaillant dans la vision des acteurs.

21 La dimension politique du travail apparaît dans cet exemple algérien comme un phénomène complexe dans lequel s'articulent et s'enchevêtrent diverses logiques, à des niveaux différents. Si l'État contribue de manière décisive à la définition des catégories du travail, du chômage et de l'emploi, à leur ordonnancement hiérarchique et sexué, voire ethnicisé, il concourt également par ses interventions ou par son absence (le « manque de contrôle » est un leitmotiv des entretiens menés en Algérie) à structurer les rapports de travail. Les logiques sociales qui s'offrent à l'observation dans les situations professionnelles ne sont cependant pas une matière inerte qui serait façonnée par l'État: elles s'élaborent dans une dynamique particulière, s'ancrent dans une histoire politique singulière. Elles ne sont en aucun cas enfermées dans un monde qui serait clos sur luimême, dans sa dimension locale ou même nationale. Les représentations que se forgent les acteurs dans le jeu de leurs interactions répondent en effet aux processus économiques, politiques ou encore idéologiques d'échelle mondiale ainsi qu'aux normes que véhicule la globalisation. Parmi ces dernières, les prescriptions de genre sont un exemple particulièrement significatif.

Les relations entre les sexes sont en effet un réceptacle des contradictions qui agitent les sociétés dans leurs rapports internes et externes, et constituent un terrain d'affrontement avec l'autre. Leur conflictualité s'exprime dans les tensions entre une supposée égalisation véhiculée par le marché - qui se révèle toujours une imposture en prétendant rendre intolérable toute forme de discrimination - et des enjeux statutaires et identitaires stimulés par l'exacerbation de la concurrence marchande et des inégalités. C'est pourquoi nous nous efforcerons maintenant de mettre en évidence les principales orientations des études articulant la question du travail et celle du genre, qui connaissent un développement remarquable.

\section{Les signifiants du sexe dans le travail}

Depuis maintenant près de deux décennies en France les facteurs de sexe se sont en effet inscrits dans les sciences sociales du travail jusqu'à constituer des domaines d'études propres évoluant vers de plus en plus d'autonomie. Le MAGE au CNRS (Marché du travail et genre), la revue Travail, genre et société s'en comptent parmi les meilleures illustrations. La thématique, au départ restreinte à l'Europe, débouche aujourd'hui sur des collaborations internationales en Amérique latine, en Asie, et un authentique rayonnement. Comme dans d'autres sous-branches spécialisées des sciences sociales, l'inspiration est venue du courant nord-américain des gender studies qui se sont développées de façon exponentielle dans le cadre de la globalisation, après la chute de l'URSS. Leur importation (Selim, 2011a) et leur implantation dans des contextes sociétaux et politiques divers donnent à voir une pluralité d'outils, d'objectifs, de cibles, de modalités de financements. Les universités sont toujours privilégiées et ce, d'autant plus que la collaboration de femmes intellectuelles est à la fois enthousiaste, productive et inventive. Sous cet angle la France peut être incluse dans une comparaison qui s'étendrait de l'Europe ex-communiste au Mexique, à la Chine, pour ne cibler que quelques régions. Le transfert cognitif, de par l'implication des actrices, offre au regard une foule de tableaux contrastés, la réappropriation d'items et des schèmes principaux, leurs redéploiements originaux, et les nouveaux éclairages qui en sont issus se diversifiant 
selon les profils historiques des microcosmes concernés. En France, lorsque les gender studies font leur entrée, la sociologie du travail - dont la revue du même nom est un éponyme exemplaire - n'est déjà plus le champ fortement structuré tant au plan méthodologique qu'idéologique qu'elle fut pendant 30 ans. Les chercheurs, dont l'affiliation marxiste est majoritaire, sont doublement ébranlés : outre que le courant antitotalitaire fait sa percée en France (Christofferson, 2009) bouleversant leurs visions, la transformation de l'organisation du travail, les nouvelles normes de management et leurs effets remettent en cause leurs propres modes d'entrée dans les usines - par l'alliance avec les syndicats - et leurs foci habituels - qualifications, postes de travail, chaîne, équipe, etc. Repensées par les chercheurs qui s'en emparent, les gender studies apportent donc dans les sciences sociales françaises du travail une nouvelle veine féconde, qui depuis ne cesse de s'amplifier.

24 Si l'on s'essaye à caractériser les études multiples qui intègrent maintenant les facteurs de sexe dans le travail, on repère cinq mots clés qui en bâtissent les armatures herméneutiques et pratiques indissociables : femmes, hommes, genre, discriminations, inégalités. Ces mots ne se situent pas dans les mêmes plateaux d'intelligibilité et c'est précisément le croisement des plateaux sélectionnés qui retient l'attention, tout particulièrement si on le confronte aux anciens dispositifs historiques de la sociologie du travail. Les termes femme/homme, leur dichotomisation affirmée dans des investigations centrées exclusivement sur des femmes serait subsumée par le concept constructiviste de genre. Mais les notions de discriminations et d'inégalités rabattent le regard sur la séparation irréfragable des destinées, marquées par les charges inesquivables de la maternité. L'action publique, l'influence sur l'espace et les politiques publiques avec lesquelles les études partagent une partie du vocabulaire, semblent dominer les approches qui ont pour horizon une forme de rétablissement de la justice entre hommes et femmes au travail dont les inégalités de traitement sont décortiquées, ou au moins une amélioration de la condition des femmes au travail, discriminées dans le marché du travail et le déroulement des carrières. Ce positionnement et ces résultats ont une légitimité certaine que vient encore conforter, s'il le fallait, leur utilité publique. De surcroit ils sont beaucoup plus en harmonie avec les dynamiques du moment, que ne le fut en son temps l'ancienne sociologie du travail, adossée aux luttes politiques et non immergée dans l'espace public, cramponnée à la classe ouvrière et aux catégorisations des travailleurs résultant des affrontements syndicaux avec le patronat. La sousdiscipline tenait alors à se distinguer de la sociologie industrielle - d'origine nordaméricaine qui se construisait sur l'idée de négociations pour mieux éradiquer conflits et «lutte des classes» - de la sociologie des organisations qui à ses yeux annihilait la dimension capitaliste de l'entreprise et de la société, de la sociologie des professions qui s'enfermait dans la singularité des métiers et de la toute récente à l'époque sociologie des entreprises qui a fait son chemin depuis.

La focale genre et travail occupe désormais une place importante dans les sciences sociales du travail en partie grâce à son inclusion active dans l'espace idéologique du genre. Les mutations conceptuelles qui l'innervent s'observent d'une manière plus générale dans les orientations actuellement majoritaires des sciences sociales mais prennent, dans le champ du travail, une tonalité particulière. Si l'on tente de mieux pénétrer dans les arcanes de ces mutations, quelques lignes immédiates de déchiffrement se découvrent dans un premier temps. La première transparaît avec acuité dans la thématique à succès, femme, travail famille (Selim, 2011b) et toutes ses déclinaisons 
conciliatrices qui pointent une hypersexuation binaire du champ social du travail en appelant à des politiques publiques réparatrices aidant les mères à mieux prendre en charge la famille pour pouvoir assumer un travail. La différence homme/femme est creusée par la double identification de la femme à la mère et à la famille et l'avenir semble aussi lourd que le présent. On est au plus loin d'un imaginaire de neutralisation des appartenances de sexe dans le travail auquel les chercheurs avaient dans les années 1970 rêvé et le destin de celles qu'on dénomme femmes semble scellé dans leur être. À un deuxième niveau, l'enterrement général des concepts de domination et d'aliénation - qui étaient fondés sur l'hypothèse de la conscience mais aussi de l'inconscient comme définition de l'humanité - remettent la contrainte du réel dans une place inaugurale et primordiale, antithétique du rôle appréhendé comme essentiel de la liberté : en d'autres mots il s'agit pour les femmes de s'adapter aux nouvelles formes de contractualisation du travail, dès lors que celles-ci leur permettent de disposer de temps hors travail pour leur progéniture. Inutile de s'affronter aux impératifs managériaux puisque précarité/ flexibilité, auto-entrepreneuriat, etc. ont certains aspects bénéfiques pour les femmes plus disponibles pour leur famille. Ainsi, exemplaire parait la revendication lancinante et largement médiatisée de réunions de travail se terminant tôt afin de permettre aux femmes de rejoindre leur foyer, si indécente serait l'idée qu'elles préfèreraient passer leur soirée avec leurs collègues! Si le travail a perdu sa centralité dans les conceptions globales - conséquence du primat spéculatif financier - pour les femmes il se voit là remarquablement décentré et redevient une occupation subalterne peu productrice de richesses, aménageable et facilement supprimable.

À un troisième niveau du triangle constitutif amputé domination-aliénation-exploitation, seul ce dernier terme apparaît souvent dans les études "genre et travail», la conservation du concept d'exploitation venant en renfort aux contours de l'être-femme, discriminée, injustement traitée, bref victime des ordonnancements du travail.

Les paradigmes de genre appliqués au travail ont donc l'intérêt majeur de mettre à jour des axiomatiques sexuées longtemps oblitérées, de dévoiler leurs effets structurant et pénalisant pour les femmes, mais dans le même moment elles véhiculent malgré elles des logiques normatives et forclusives : le réel mis à plat est l'objet d'une hypostase et le pivot de recherches dont les analyses s'enferment volontairement dans la description des faits, ce qui pousse, par ailleurs, à la reprise en chœur du terme d'ethnographie, comme garant de scientificité et d'objectivité (Bazin, Selim, 2002). Ce réalisme cognitif qui s'inscrit en opposition avec une lecture des phénomènes sociaux en termes de production - et aussi de contradictions - est largement partagé aujourd'hui dans les sciences sociales mais ses connotations dans la matrice "genre et travail » sont spécifiques. Le constructivisme du genre s'évanouit tout comme la production des rôles sexués et les femmes prennent place aux côtés des « minorités visibles », « ethniques » et « raciales », toutes discriminées dans les champs du travail et de fait blessées dans leur subjectivité réduite à leur appartenance originelle. Ce constat réaliste débouche sur une demande de réorganisation, d'ajustements qui prend pour cible la position des femmes (et des autres) et non les structures de travail elles-mêmes dont la transformation radicale est aujourd'hui quasi impensable. Le care dans "genre et travail» optimise - si l'on peut dire - ces orientations : la femme domestique, procurant des services aux déficients, est en effet une travailleuse qui vend ses «capacités féminines naturelles » de dévouement contre rémunération. Doublement liée par ses dispositions intérieures et ses relations professionnelles personnalisées, parangon antérieur de l'aliénation, elle est aujourd'hui 
redécouverte comme symptôme des transformations économiques majeures qui ont promu les services et délocalisé la production et modèle du souci de l'autre et des faibles.

\section{Des normes articulées}

Tout en s'appuyant sur les acquis des études " genre et travail », il apparaitt nécessaire de produire et de penser les terrains de façon à permettre de s'écarter des forclusions normatives esquissées. Saisies dans leurs rapports imaginaires avec l'État et le monde global, et pas seulement replacées dans les espaces de contraintes familiales et de travail - fussent-ils bienheureux dans leurs assignations - les femmes que nous rencontrons et avec lesquelles nous nous entretenons sur leur trajectoire, ont l'opportunité d'élaborer d'une façon inédite le sens de leur vie face à un autre - l'ethnologue - appelé-e à disparaître de leur champ de vision. Le vide apparent de cet autre, son absence future initient - parfois, souvent, dans le meilleur des cas - une demande de parole qui est un exercice de réflexion autoscopique potentiellement douloureux, bouleversant. Insatisfactions, trouées regrets, accusations, consternations, résignations parsèment ces anamnèses ponctuelles dans lesquelles s'offre à l'écoute l'imbrication étouffante des dépendances personnelles et des coercitions objectives qui ont façonné l'individu, contemplant ses échecs, ses ratages pour prendre d'autres voies.

La Chine offre de ce point de vue un tableau particulièrement exemplaire. La politique de l'enfant unique dans un contexte de croissance capitaliste exponentielle initiée par le régime communiste monopoliste a eu des effets contradictoires sur les représentations et les pratiques de la dualité sexuelle. La domination s'en est vue outrancièrement accrue dans sa visibilité et son incontestabilité à plusieurs niveaux; tout d'abord la volonté d'engendrer un garçon a eu pour conséquence des infractions multiples à la loi, si l'on en juge par nos enquêtes menées à Canton, où en dépit des amendes élevées les couples procréent jusqu'à obtenir le garçon tant attendu. Pour les femmes quelle que soit leur classe sociale et leur niveau d'éducation, la pression de la parentèle est énorme et anxiogène, beaucoup craignant que leur mari ne partent acheter un héritier mâle à la campagne par contact direct ou indirect avec un "ventre rural ». Lorsque les jeunes femmes sont fonctionnaires ou occupent un emploi important, source de revenus nécessaires au ménage, le dilemme est terrible entre perdre leur travail, grever lourdement la réputation de leur unité de travail, pénaliser leurs collègues par un second enfant, ou voir leur mari se dispenser de leur matrice et s'en procurer à faible coût une autre. Dans tous les cas l'intériorisation d'une déficience personnelle, la culpabilité, l'infériorisation de soi pour cause de naissances de plusieurs filles à la place du garçon tant désiré affaiblit terriblement la position des femmes dans leur champ de parenté, comme de travail. En effet la prépondérance des hommes en haut des hiérarchies professionnelles qu'il s'agisse des entreprises ou des universités, revêt une dimension politique ostensible du fait même qu'à ces niveaux supérieurs de commande les acteurs sont des membres du Parti au pouvoir. La légitimation étatique de la domination masculine qui se voit de cette façon mise en œuvre accentue les rapports d'autorité et de maitrise et donne aux femmes le sentiment qu'il est inutile de tenter de se battre. Naturalisé, politisé, étatisé, le pouvoir des hommes offre à l'observation des formes d'expansion totalisantes que viennent encore renforcer les logiques statutaires actuelles fondées sur une consommation ostentatoire et la réalisation de la " famille harmonieuse " dans une société aux tensions sociales pacifiées. L'efficacité des normes parait dans ce 
contexte irréfragable et les femmes montrent leur incorporation remarquable dans leurs efforts inouïs mais pour les concrétiser dans leurs vies. Etre de bonnes épouses, bellesfilles, mères passe largement avant leur travail. Celles qui s'écartent de ce modèle risquent de fait de prendre la voie de la dissidence, la réfutation de la norme de la domination masculine se confondant avec celle de l'État-parti dont les représentants sont des hommes.

Le risque de devenir «une femme restante » parfait ce paysage par la honte qu'elle projette sur le sujet ; marchandise avariée, à jeter, la femme célibataire de plus de trente ans est jugée aujourd'hui par l'État-parti un véritable «problème social». Ces « femmes restantes » qui n'ont pas trouvé « l'âme sœur » sont le plus souvent hautement diplômées et montrent des choix de carrière privilégiés. Inversant la structure paradigmatique de la domination sexuée dans l'hypothèse où elles épouseraient des inférieurs, elles sont l'exacte antithèse des « hommes restants » qui peuvent trouver des femmes toujours plus en bas de l'échelle sociale, ne serait-ce qu'en allant les acheter au Vietnam par exemple. La nature du régime autoritaire de la Chine - avec à sa tête un État-parti qui se modernise mais dans le même moment redoute de plus en plus les remises en cause de la " société civile » en germe et en 2012 resserre son pouvoir par la répression - a ainsi pour conséquence d'ontologiser les rapports de domination dans le cadre de la dualité sexuelle et d'en produire une justification substantielle dans tous les champs sociaux et en premier lieu celui du travail, intimement articulé pour les femmes à leur mode d'existence dans l'alliance et la parenté. Leader de la globalisation dans le monde actuel, la Chine offre ainsi à la réflexion, un visage paradoxal où le désir de s'affranchir du joug de l'État est manifeste dans une partie grandissante de la population, mais où l'émancipation de la moitié du ciel n'est plus à l'ordre du jour.

D'une manière générale, dans une perspective comparative, la/les normes ressortent des paysages sociaux comme des productions tout à la fois intérieures et extérieures qui peuvent être fuies, déviées, étayées, nourries, consolidées, etc. Leur implacabilité s'étoffe et se dissout dans le même moment. "Si j'avais été un homme " peut-être lâché comme une conclusion suspendant le temps dans des pays aussi différents que le Vietnam, le Bangladesh, l'Ouzbékistan, le Laos, la Chine, sans oublier la France. L'inscription dans le travail devient alors un kaléidoscope ouvrant les fenêtres des autres sphères d'inclusion et l'espace de travail se révèle un lieu de cristallisation des contradictions éclatées qui agitent l'ensemble de la société et interrogent l'État, sa figure imaginaire autant que l'action publique. C'est en effet - en dernière instance - l'État qui décide du sexe d'un individu, marqué dès sa naissance, sur ses documents d'identité lui permettant d'échapper à sa société, de parcourir le monde et de nier symboliquement son appartenance de sexe ainsi qu'en témoignent les nombreux récits des femmes qui à différentes époques se déguisèrent en hommes pour simplement voyager. C'est encore à la puissance étatique qu'il faut réclamer l'agrément d'un changement de sexe. Notons à ce propos que des revendications émergent pour demander que le sexe n'apparaisse plus sur les documents d'identité. En revanche, les États - dans le cadre de la globalisation du capitalisme - ont perdu leur pouvoir de légiférer avec efficacité sur les espaces de travail livrés aux règles du profit financier. 


\section{Conclusion}

Dans le domaine du travail, comme dans d'autres champs sociaux, la norme de genre a souvent conduit à une scotomisation de l'État et à une occultation du politique dans les fonctionnements sociaux et dans les imaginaires qui contribuent à les rendre efficients. Il importe de réaffirmer le caractère politique des sexuations des rapports sociaux et ce en particulier dans le travail où viennent se confronter et s'enrichir des approches objectivistes mais aussi d'autres centrées sur la subjectivité des acteurs. La complémentarité de ces approches renvoie d'une certaine manière aux prises de parole et aux analyses qui ont éclos dans le cadre du mouvement de libération des femmes dont les commémorations ont eu lieu en 2010 sur un mode parfois très politisé en direction d'une allophobie encouragée par l'État prenant comme argument le «voile»: celles-ci proclamaient pourtant une implication politique globale - sur le corps, la conscience, l'inconscient, le travail domestique non rémunéré et salarié, etc. - et appelaient à la décrypter pour mieux secouer les jougs qu'elle concrétise (Storti, 2012). Que ce soit dans une période de révolte politique que soient pointées les déterminations du politique paraît cohérent dans cette perspective; corollairement dans la conjoncture présente d'affaiblissement des États sous surveillance des agences de notation et de raréfaction des emplois, on ne saurait être surpris que les appartenances identitaires exaltées, dont celles de sexe, s'érigent dans l'autonomie et l'effacement des constructions politiques sur lesquelles elles reposent. Que le sexe devienne l'objet d'une revendication le posant comme un travail marchand comme les autres semble en outre en continuité avec les idéologies dominantes établissant la liberté absolue du sujet et des échanges dans lesquels il s'engage. La posture abolitionniste et répressive n'apporte la contradiction que pour mieux légitimer et ancrer son apparent contraire : tout comme la figure de la femme «voilée », celle de la prostituée est perçue comme victime de la domination et de l'exploitation maximale, tout en faisant écueil aux injonctions normalisées d'égalisation et d'émancipation, d'où découle l'acharnement à la marginaliser et à l'effacer de l'espace public - et de celui du travail.

En revanche, dans le champ social du travail, le risque est peut-être perceptible que le genre, sectionné et coupé, favorise dans l'imaginaire l'éloignement des enjeux essentiels des rapports socioéconomiques s'étayant sur le politique. La réduction hyperbolique du coût du travail se donne à voir aujourd'hui comme la clé de construction du capitalisme globalisé; elle parcourt tous les discours de légitimation des gouvernements, obligés d'endosser cette pseudonorme de gouvernance économique de plus en plus hégémonique. Ainsi cette réduction du coût du travail inscrit l'État et les rapports sociaux de sexe dans un triangle critique révélateur des processus: elle soumet l'État, exacerbe les affrontements entre les hommes et les femmes et démultiplie dans l'imaginaire, le symbolique et le réel tous les plateaux de la domination: la domination étatique, la domination masculine, l'exploitation du travail, comme le montrent les exemples algérien et chinois. 


\section{BIBLIOGRAPHIE}

Actes de la recherche en sciences sociales, 1996 : Les nouvelles formes de domination dans le travail, $\mathrm{n}^{\circ} 114$.

Althabe G. (1963) : Étude du chômage à Brazzaville. Paris, Cahiers ORSTOM - Série sciences humaines, I, 4.

Arborio A.-M., Cohen Y., Fournier P., Hatzfeld N., Lomba C., Muller S. (2008) : Observer le Travail. Paris, La Découverte.

Bazin L., Gibb R., Selim M. (2007) : « Nationalisation et étatisation des identités dans le monde contemporain », Journal des anthropologues, hors-série « Identités nationales d'État » : 7-35.

Bazin L., Selim M. (2002) : « Ethnographie, culture et globalisation. Problématisations anthropologiques du marché », Journal des anthropologues, 88-89 : 269-305.

Beaud S., Pialoux M. (2005) : Retour sur la condition ouvrière. Paris, Fayard.

Christofferson M. (2009) : Les intellectuels contre la gauche, l'idéologie antitotalitaire en France (1968-1981). Marseille, Agone.

Cooper F. (1996) : Decolonization and African Society. The labor question in French and British Africa. Cambridge, Cambridge University Press.

Dejours C. (2000) : Souffrance en France. Paris, Seuil.

Dressen M., Durand J.-P. (dir) (2011) : La violence au travail. Paris, Octave éditions.

El-Kenz A. (dir.) (1987) : L’Algérie et la modernité. Dakar, éd. CODESRIA.

Guérid, D. (2007) : L'exception algérienne. La modernisation à l'épreuve de la société. Alger, Casbah éditions.

Lautier B., Miras C. de, Morice A. (1994) : L'État et l'informel. Paris, L'Harmattan.

Selim M. (2011a) : «L'importation des gender studies à Canton, usages personnels, collectifs, politiques », in Castelli B., Hours B. : Enjeux idéologiques de la globalisation en sciences sociales. Paris, L'Harmattan : 213-237.

Selim M. (2011b) : « La face cachée des femmes-outils », L'Homme et la société, 176-177 : 253-266.

Storti M. (dir.) (2012) : Le féminisme à l'épreuve des mutations géopolitiques. Paris, éditions iXe.

\section{NOTES}

1. Cf. aussi le festival « Filmer de travail », Poitiers, depuis 2010.

2. Cf. Actes de la recherche en sciences sociales (1996); Dressen, Durand (2011).

3. Comme c'est d'ailleurs le cas dans la définition même dont se dotent les partis de gauche, notamment le Parti socialiste en France : dans le socle de valeurs auquel se rattache le parti, la référence ouvrière disparaît tandis que la mention à l'économie de marché fait son apparition.

4. La harga (du verbe brûler - les frontières, mais aussi ses papiers, sa vie...) est la fuite des jeunes (chômeurs, sous-employés ou sans perspectives d'avenir) à travers la Méditerranée pour 
gagner l'Europe en embarcations de fortune. C'est un phénomène qui capte l'attention et l'imaginaire dans l'Algérie contemporaine, même s'il demeure probablement peu signifiant numériquement.

5. Cf. les travaux de Mehdi Larbi.

6. De nombreuses recherches sociologiques algériennes dans les années 1980 ont montré comment le travail est au centre du «contrat social » populiste mis en place par le pouvoir algérien à l'indépendance (Saïd Chikhi, Ali El-Kenz, Djamel Guérid, Mohamed Mebtoul...). Ces travaux n'ont pas été actualisés à partir de la mise en place du plan d'ajustement structurel (1994) et la guerre entre l'État et les factions islamistes des années 1990. On se réfère ici plus particulièrement à El-Kenz (1987), Guérid (2007).

INDEX

Mots-clés : critique du travail, correspondance, artistes, socialisme vert, travailleurs culturels, Wertkritik, Gorz André, dialogue, Vincent Jean-Marie, écologie, modèles alternatifs, Marx Karl, penser le Capital, ethos du travail, Eglise Orthodoxe, salariat, domination

\section{AUTEURS}

LAURENT BAZIN

CNRS-CLERSÉ

MONIQUE SELIM

IRD, UMR 201 